\title{
Spatial filtering in edge-emitting lasers by intracavity chirped photonic crystals
}

\author{
S. Gawali, ${ }^{1, *, \dagger}$ J. Medina, ${ }^{1, \dagger}$ D. Gailevičius ${ }^{2,3}$ (D) V. Purlys,${ }^{2,3}$ (D) G. GArre-Werner, ${ }^{1,4}$ (i) \\ C. Cojocaru, ${ }^{1}$ (i) J. Trull, ${ }^{1}$ M. Botey, ${ }^{1}$ R. Herrero, ${ }^{1}$ J. Montiel-Ponsoda, ${ }^{4}$ and \\ K. STALIUNAS ${ }^{1,2,5}$ \\ ${ }^{1}$ Universitat Politècnica de Catalunya (UPC), Physics Department, Rambla Sant Nebridi 22, 08222, Terrassa, Barcelona, Catalonia, Spain \\ ${ }^{2}$ Vilnius University, Laser Research Center, Saulètekio al. 10, LT-10223, Vilnius, Lithuania \\ ${ }^{3}$ Femtika, Saulètekio al. 15, LT-10224, Vilnius, Lithuania \\ ${ }^{4}$ Monocrom S.L, Vilanoveta, 6, 08800, Vilanova i la Geltrú, Catalonia, Spain \\ ${ }^{5}$ Institució Catalana de Recerca i Estudis Avançats (ICREA), Passeig Lluís Companys 23, 08010, Barcelona, Catalonia, Spain \\ *Corresponding author: sandeep.babu.gawali@upc.edu
}

\begin{abstract}
In this work, we report an experimental and numerical study of the intracavity spatial filtering in edge-emitting lasers using a chirped photonic crystal $(\mathrm{PhC})$ as the filtering element in the near-field domain. We provide a comprehensive analysis of the near-field PhC filtering scheme and compare it to conventional spatial filtering using a variable width slit in the far-field domain. Using a two-dimensional chirped $\mathrm{PhC}$ as a spatial filter, we experimentally demonstrate a brightness enhancement by a factor of 1.3, considering an edge-emitting laser with a $1.5 \mathrm{~mm}$ cavity length, consistent with a numerical prediction of brightness enhanced by a factor of 1.7. The experimental results are theoretically confirmed by numerical integration of a spatio-temporal model of the edge-emitting laser. Furthermore, numerical results show that brightness can be further increased over a factor of 2 , applying optimized spatial-filtering elements at both the front and rear facets of the lasers.
\end{abstract}

\section{INTRODUCTION}

Nowadays, semiconductor edge-emitting lasers, due to their compactness, reliability, and affordability, represent a competitive light source for a wide range of fields such as medicine, telecom, material processing, consumer electronics, and pumping solid-state lasers, among others. However, while still playing an important role, in high-power regimes they suffer from poor beam quality along the junction direction (slow axis). The light is emitted from a rectangular aperture having dimensions of few micrometers along the fast axis, vertical direction $(y)$ in Fig. 1(a), and 50-400 $\mu \mathrm{m}$ in the slow axis, horizontal direction $(x)$ in Fig. 1(a) [1]. Since the beam is tightly confined along the fast axis, it diverges very strongly after exiting the aperture, but can be collimated using a cylindrical lens due to an almost single-transverse-mode radiation in this direction. In contrast, the beam along the slow axis is less confined leading to multimode emission and to a problematic collimation along this direction. In the far field, the beam has an elliptical shape with degraded spatial quality that prevents coupling to optical fibers with a small core diameter and low numerical aperture (NA). The absence of any intracavity mode selection mechanism is the reason for the poor beam quality in such lasers. Moreover, for most of the applications, laser diodes are typically operated at a high current level where their electro-optical efficiency is the highest. However, hard pumping induces non-linear effects that excite more spatial modes degrading the beam quality of the laser even more.

There are several demonstrated techniques to improve the beam quality in edge-emitting lasers, such as conventional spatial filtering using off-axis feedback [2], external cavity [3-5], tapered geometry [6], and evanescent spatial filtering [7]. High spatial quality single-mode beams can also be obtained by restricting the width of the active region of a ridge waveguide [8]. All these mentioned methods can improve the beam quality problem by compromising either the laser compactness or its emission power, so they are not suitable for compact and high-power microlasers. An alternative approach consists of modulating the active medium [9], yet it is technologically challenging to be implemented. In order to overcome these limitations, new solutions are needed.

Photonic crystals (PhCs) can be a promising solution due to their compact nature. In one experimental study, it was demonstrated that a high spatial quality beam can be obtained at watt-level power by incorporating the $\mathrm{PhC}$ inside the laser cavity of a vertical-cavity surface-emitting laser [10]. In our work, the $\mathrm{PhCs}$ will be used as compact spatial filters to improve the beam 
quality in edge-emitting lasers. The idea of spatial filtering using $\mathrm{PhC}$ has been proposed [11] and experimentally demonstrated $[12,13]$ in transmission schemes. The advantage of the $\mathrm{PhC}$ filtering is that it works in a near-field domain, contrary to conventional filters acting in the far-field domain. In an ideal case, designing semiconductor lasers with high spatial beam quality would require placing these compact spatial filters fixed in a monolithic implementation between the front facet and the cavity mirror, avoiding the need for extra space to affect the beam in the far field. PhCs offer advantages in terms of their size to miniaturize the device, for instance, in microlasers. At the current technological level, the use of PhCs in a monolithic implementation still represents a challenging task. To mimic the desired configuration, a simplified approach is followed, in which the $\mathrm{PhC}$ is placed at the accessible near-field plane in an extended cavity. The action of the $\mathrm{PhC}$ in the near-field area of extended resonator is thus equivalent to its action in the monolithic configuration inside the laser microcavity. In a previous study, the first proof of spatial filtering in edge-emitting lasers using an intracavity $\mathrm{PhC}$ was provided [14].

In this work, we present a detailed numerical and experimental study of $\mathrm{PhC}$ spatial filtering in edge-emitting lasers. As a first step, we compare the results of such $\mathrm{PhC}$ spatial-filtering schemes with a conventional filtering in the far-field domain using an intracavity slit. Along with spatial filtering, the brightness enhancement is the main motivation of this work. We numerically analyze and experimentally demonstrate beam profile improvement by measuring the beam quality factor, $M^{2}$, and simultaneous brightness enhancement of the emitted beam. We also propose an optimized scheme to improve the brightness enhancement by using the multiple filtering elements at the front and rear laser facets.

\section{EXPERIMENTAL SETUP}

The spatial-filtering performance of chirped PhCs in edgeemitting lasers is explored using an extended cavity scheme. The actual experimental setup consists of an anti-reflection (AR) coated laser, microcylindrical fast axis collimator (FAC), two pairs of cylindrical lenses to create conjugate planes, and a feedback mirror. The front facet of the laser is AR coated $(R<0.01 \%)$, while the back facet has reflectivity of $95 \%$. The active length of the laser diode chip is $1.5 \mathrm{~mm}$ while the transverse width is $400 \mu \mathrm{m}$. The operating wavelength was $970 \mathrm{~nm}$ at a driving current of $3 \mathrm{~A}$ and the total emitted power of $1.24 \mathrm{~W}$ in continuous wave $(\mathrm{CW})$ mode. Throughout the experiment, all the measurements were performed by driving the laser in pulsed mode $(50 \mathrm{~Hz}, 25 \%$ duty cycle).

The high divergence emission along the fast axis is collimated using a high NA (0.8) FAC microlens, with a focal length of $590 \mu \mathrm{m}$. A double $4-f$ plano-convex cylindrical lens system, acting along the slow axis, is used to create two conjugate planes of the emitter facet plane. The lenses $\mathrm{L}_{1}-\mathrm{L}_{2}$ and $\mathrm{L}_{3}-\mathrm{L}_{4}$ are arranged in a confocal arrangement forming the self-imaging cavity, which returns the same field to the same position after one round trip. The focal length of lenses $\mathrm{L}_{1}-\mathrm{L}_{4}$ is $f=50 \mathrm{~mm}$, and all the lenses used are AR coated for near-infrared wavelength. The chirped $\mathrm{PhC}$ filter is placed at the first conjugate plane, plane B in Fig. 1(c), and the laser cavity mirror is located at the second conjugate plane, plane D in Fig. 1(c). The reflectivity of the mirrors used varies between $4 \%$ and $8 \%$ in one side and having an AR coating on the other side. Lasing at the lowest threshold current is achieved by carefully tuning the mirror. The total length in this extended cavity configuration is around $400 \mathrm{~mm}$. This setup allows for the characterization of the effect of spatial filtering using either the chirped $\mathrm{PhC}$ in plane $\mathrm{B}$ or a conventional intracavity filter slit at the Fourier plane, plane $\mathrm{C}$ (a)

(c)

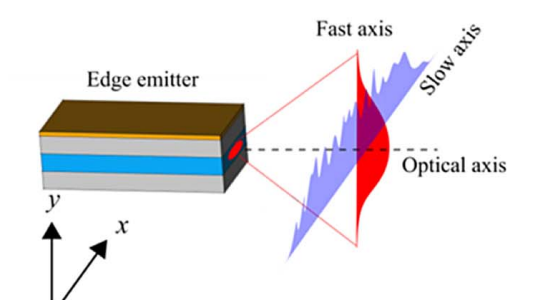

(b)
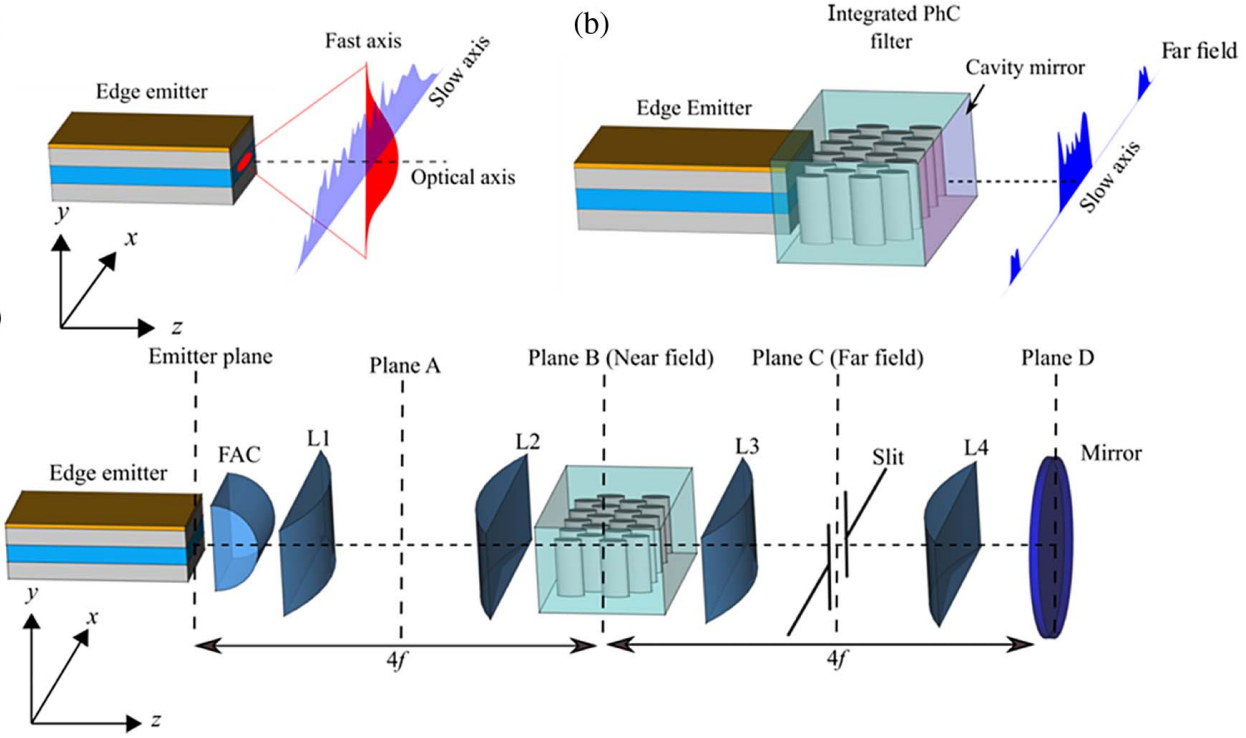

Fig. 1. (a) Emission from an edge-emitting laser with high divergence (but single mode) along fast axis $(y)$ and lower divergence multimode emission along the slow axis $(x)$. (b) Monolithic integrated $\mathrm{PhC}$ filtering scheme in compact cavity configuration. (c) The experimental setup of the laser in the extended cavity spatial filtering in a double $4-f$ configuration. $\mathrm{FAC}$ is the fast axis collimator, lenses $\mathrm{L}_{1}-\mathrm{L}_{4}$ are plano-convex cylindrical lenses acting in the horizontal (slow axis) plane with focal length $f$. Each $4-f$ imaging system performs a Fourier transform, followed by an inverse Fourier transform. Planes B and D are conjugate planes of the emitter facet plane, while planes A and C are the corresponding Fourier planes. The mirror on plane $\mathrm{D}$ has a variable reflectivity (from $4 \%$ to $8 \%$ reflection). 
in Fig. 1(c), in the same setup. The improvement of the output laser beam is studied by measuring the power, the spectrum, the $M^{2}$ factor, and the laser near- and far-field profiles.

The beam profiles at the output of the laser (near field) or at the focal plane of an external lens (far field) are recorded by imaging the profiles into a CCD camera with proper magnification. The $M^{2}$ factor is measured focusing the beam by a $100 \mathrm{~mm}$ focal length plano-convex lens and imaging the beam transverse distribution in a CCD camera (Spiricon SP620U) at different planes (around 15 data points) along the propagation axis over a distance much larger than the Rayleigh range. The $4 \sigma$ (second-moment) beam diameter is measured which defines four times the standard deviation of the spatial distribution of the beam intensity profile evaluated in the transverse direction. The obtained data is fitted with the beam propagation equation to accurately measure the $M^{2}$ value. This measurement together with the output power is used to obtain the corresponding brightness $B=\frac{P}{\lambda^{2} M_{x}^{2} M_{y}^{2}}$, where $P$ is the output power, $\lambda$ is the laser wavelength, and $M_{x}^{2}$ and $M_{y}^{2}$ are the corresponding $M^{2}$ factors along the horizontal and vertical directions, respectively.

\section{NUMERICAL MODEL FOR THE EDGE-EMITTING LASERS}

With the aim of numerically simulating the edge-emitting semiconductor lasers, we present a simplified model, which includes spatio-temporal evolution of the intracavity field and carriers. Edge-emitting lasers are generally described by stationary models for the electromagnetic fields and carriers [15] and also by simplified mean field models including temporal evolution [16]. For a precise description, a complete model is used by solving Maxwell's equations for the forward/backward fields propagating within the cavity, together with Bloch equations for the carrier's inversion [17]. Here, we develop a different approximation, by separately integrating the field propagation in space and the slow variation of carrier inversion in time [18]. This simplified model, which takes advantage of the different time scales of the optical field evolution (roundtrip is of the order of picoseconds) and relaxation of the inversion (lifetime in the order of nanoseconds), turns out to be much faster as compared with the complete model [16], and it has provided reasonable results for amplifiers and lasers.

In our method, every integration step combines the field propagation in one-cavity round trip assuming constant carriers and, subsequently, the temporal integration of carriers considering a constant field. The temporal evolution of the electromagnetic field, composed by the forward, $A^{+}$, and backward, $A^{-}$, fields and the two-dimensional distribution of carriers, $N$, is modeled by the following equations:

$$
\begin{gathered}
\pm \frac{\partial A^{ \pm}}{\partial z}=\frac{i}{2 k_{0} n} \frac{\partial^{2} A^{ \pm}}{\partial x^{2}}+s[(1-i h) N-(1+\alpha)] A^{ \pm}, \\
\pm \frac{\partial N}{\partial t}=\gamma\left(-N-(N-1)|A|^{2}+p_{0}+D \nabla^{2} N\right),
\end{gathered}
$$

where $|A|^{2}=\left|A^{+}\right|^{2}+\left|A^{-}\right|^{2}, k_{0}$ is the wavevector, $n$ is the refractive index, $s$ is interaction parameter inversely proportional to the light matter interaction length, $h$ is the Henry factor (linewidth enhancement factor of the semiconductor), $\alpha$ corresponds to losses, $\gamma$ is the carrier's relaxation rate, $p_{0}$ is the pump, and $D$ is the carrier diffusion. The material polarization of the semiconductor is adiabatically eliminated, as usual for class B lasers.

The system of Eq. (1) is completed by the boundary conditions, where the field components, $A^{+}$and $A^{-}$, are related by the corresponding relations: $A^{-}(x, z=L, t)=r_{L} A^{+}$ $(x, z=L, t) ; A^{+}(x, z=0, t)=r_{0} A^{-}(x, z=0, t)$, where $L$ is the laser resonator length and $r_{0}$ and $r_{L}$ are the corresponding reflection coefficients of the cavity mirrors at $z=0$ and $z=L$, respectively, see Fig. 2. The delay of the fields in the feedback is
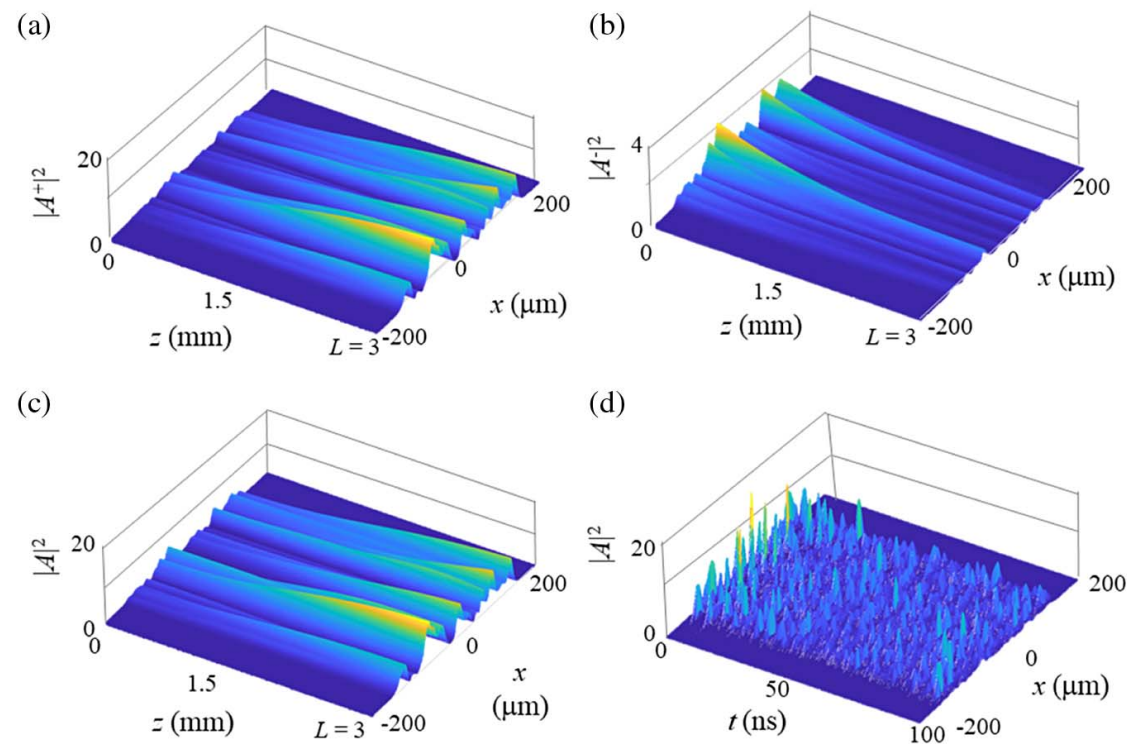

Fig. 2. Intensity of the fields within the laser for (a) forward field intensity, (b) backward field intensity, (c) total field, and (d) temporal evolution of the output intensity. Integration parameters: $p_{0}=2.0$, width $=400 \mu \mathrm{m}$, length $=1500 \mu \mathrm{m}, \alpha=0.1 \mu \mathrm{m}^{-1}, h=2.0, s=0.04 \mu \mathrm{m}^{-1}, k_{0}=$ $2 \mu \mathrm{m}^{-1}, D=0.03 \mathrm{~cm}^{2} / \mathrm{s}$, and $n=3$. 
not taken into account (as in Lang-Kobayashi model [19]), due to slowness of the population inversion.

We preliminarily analyze an edge-emitting laser with the parameters corresponding to an experimentally used laser, as proof of the numerical model, before considering the $\mathrm{PhC}$ filtering effects. The laser is modeled by the system of Eq. (1), calculating a forward propagating field, $A^{+}$, see Fig. 2(a), a backward propagating field, $A^{-}$, Fig. 2(b), and total field, $A$, see Fig. 2(c). The spatio-temporal integration exhibits both inhomogeneous and multimode spatial and unstable temporal behaviors see Figs. 2(c) and 2(d). The sufficiently good agreement with the experiment results permits one to use the model for the spatial-filtering simulation.

\section{SPATIAL FILTERING BY AN INTRACAVITY SLIT}

We study first the conventional spatial filtering using an intracavity slit positioned in the far-field domain. A well-calibrated slit with variable width and incremental step size of $0.5 \mathrm{~mm}$ centered at the optical $x$-axis is used for the experiment. While the spatial-filtering technique can be implemented outside the cavity in a single transmission scheme, the resulting power reduction for such a scheme does not bring any enhancement in brightness. The slit acts in the slow axis by laterally blocking the beam and, when placed at the Fourier plane, plane $\mathrm{C}$ in Fig. 1(c), serves as a spatial filter that introduces strong losses to higher-order transverse modes. The slit has a variable width, ranging from $6.5 \mathrm{~mm}$ to $1 \mathrm{~mm}$ corresponding to an angular transmission range between $65-10 \mathrm{mrad}$. As we start to close the slit, the higher-order transverse modes are suppressed, and for a slit width of $1 \mathrm{~mm}$, only the lowest-order transverse modes are allowed to lase. The $M_{x}^{2}$ of the unfiltered laser increases from 33-62 with an increase in pump current from 2-5 A. Since the aim of this work is to increase the brightness when filtering out the most divergent modes, we also define the relative brightness as the ratio between the brightness of the emitted beam with spatial filtering (either with the slit or with the $\mathrm{PhC}$ ) and brightness without any filtering $\left(B_{o}\right)$ scheme: $B_{\text {filtered }} / B_{o}$.

As expected, the output power is reduced when reducing the slit width, but the simultaneous reduction of the $M^{2}$ factor allows for enhancing the beam quality and the beam brightness. In addition, we explored the role of different parameters, in particular, the effect of the reflectivity of the feedback mirror and of the pump current, on the filtering performance of the edge-emitting laser. We have used three different reflectivities of the feedback mirror ( $4 \%, 6 \%$, and $8 \%$ ). We limit the reflectivity to $8 \%$ to avoid the possibility of causing catastrophic optical mirror damage to the laser facet [20]. By increasing the reflectivity of the feedback mirror, different effects are observed: the optical load at the out-coupling facets increases, the photon density is enhanced within the cavity, and the transverse far-field profile also changes because of the excitation of higher-order modes, whereas the near-field pattern remains unchanged. For the $4 \%$ reflectivity mirror, the threshold current is $1.3 \mathrm{~A}$, and the output optical power is $0.31 \mathrm{~W}(1.24 \mathrm{~W}$ in $\mathrm{CW}$ mode) at 3 A (2.3 times the threshold intensity, $I$ th) measured in pulsed mode with a repetition rate of $50 \mathrm{~Hz}$ and a duty cycle of $25 \%$, while for the $8 \%$ reflectivity mirror, the threshold current drops to $1.2 \mathrm{~A}$ giving an optical power $0.36 \mathrm{~W}(1.44 \mathrm{~W}$ in $\mathrm{CW})$ for same pump current. The wavelength peak shifts toward the right-hand side by $1.2 \mathrm{~nm}$ when changing the reflectivity of the mirror from $4 \%$ to $8 \%$ at $3 \mathrm{~A}$ of pump current. The maximum current applied to our edge-emitting laser was $5 \mathrm{~A}$.

In the numerical model, we add the slit as a transverse wavenumber cutoff filter in the Fourier space, positioned close to the front mirror. The cutoff wavenumber that simulates the slit goes from $0.25-0.5 \mu \mathrm{m}^{-1}$, which corresponds to a divergence from $70-10 \mathrm{mrad}$.

Figure 3 shows the dependence of the transmitted output power as a function of the width of the variable slit for different pumping currents in the experiments (a) and numerical simulations (b). The maximum power is recorded with no slit inside the extended cavity, decreasing as the width of the slit is reduced. Similar behavior is observed both in simulation and experiment.

By increasing the pump current, the changes in the beam are better distinguished in the far field, while the near field always presents strongly modulated and essentially unaltered profiles. The simulated output profiles are obtained by averaging thousands of integration round-trips to be compared with the experimental ones. Even though experimental and simulated far fields show some discrepancies, these could be attributed to the model simplification [21]. As we increase the pump current, the output optical power increases along with the $M^{2}$-factor due to excitation of more spatial modes. To evaluate the effect of slit filtering, we experimentally measured and numerically calculated the increase in the brightness of the output beam for lasers with the same dimensions and analog working situations. We observe that the absolute brightness continues to increase when pump current is increased, however, the relative brightness decreases
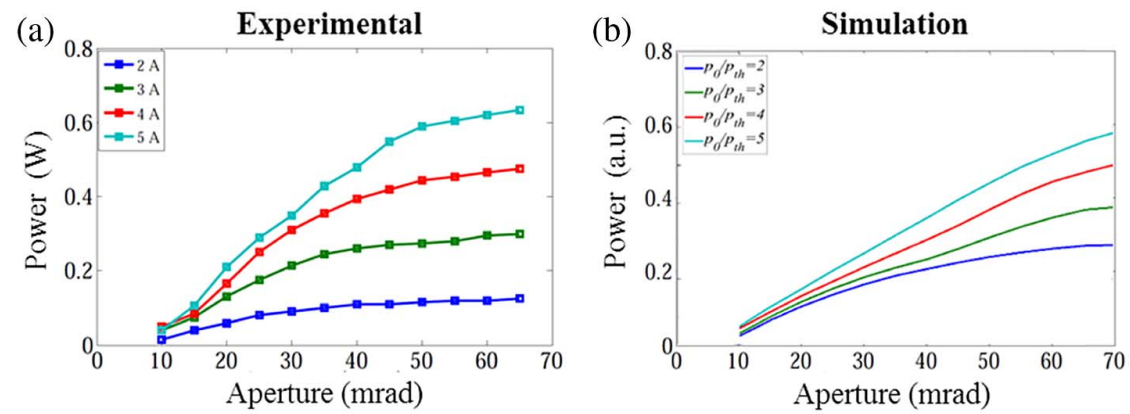

Fig. 3. Dependence of the output power with the slit width. (a) Experimental measurement and (b) numerical simulation. In numerical simulation, the pump current is normalized to threshold current. Experimental powers correspond to pulsed emission ( $50 \mathrm{~Hz}, 25 \% \mathrm{duty}$ cycle). 

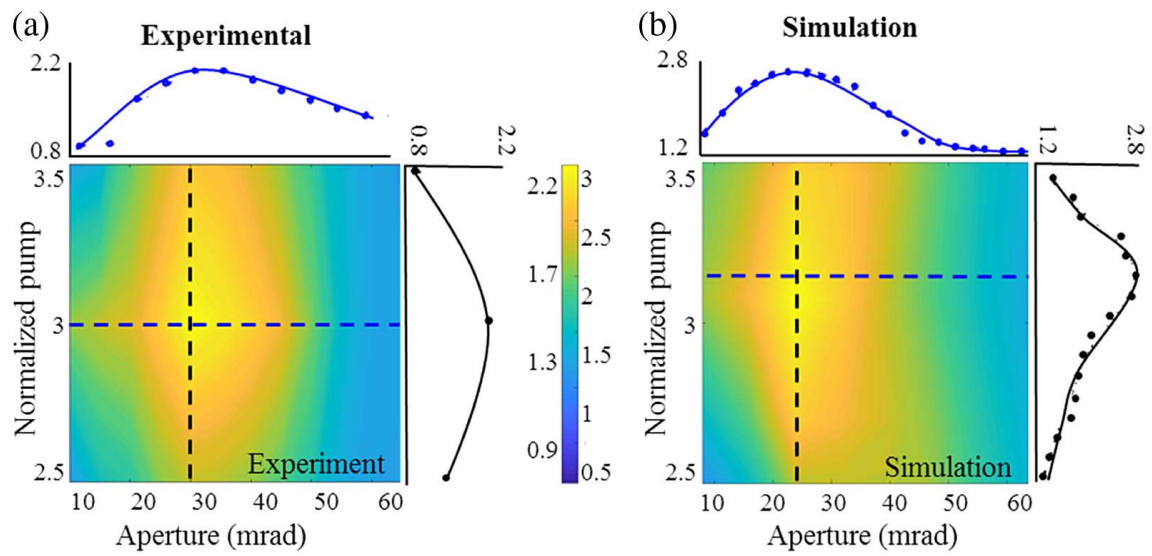

Fig. 4. Relative brightness as a function of the slit aperture and normalized pump current. The pump current is normalized to the threshold current. Comparison of the (a) experimental and (b) numerical results of relative brightness for $1.5 \mathrm{~mm}$ cavity length and $400 \mu \mathrm{m}$ width edge-emitting laser. Both figures provide the cross-sections at the maximum relative brightness following the dashed lines. The reflectivity of feedback mirror is $4 \%$ in this case. The lines are a guide for the eye.

after an optimum value is achieved. This maximum value corresponds to the aperture of $30 \mathrm{mrad}$ at a normalized pump current of 3 (normalizing to the threshold current). Similar results were observed by changing the reflectivity of the laser cavity mirrors from $4 \%-8 \%$. The numerical results are obtained by integrating Eq. (1), including a slit filter in the front facet. The experimental results in Fig. 4(a) and the numerical simulations in Fig. 4(b), show the same tendency. The maximum relative brightness is of the order of 2.2 in both cases and occurs at similar apertures, around $25 \mathrm{mrad}$ and for a normalized pump current close to 3 .

\section{PHOTONIC CRYSTAL FABRICATION AND CHARACTERIZATION}

The used $\mathrm{PhC}$ is a compact two-dimensional periodic structure inscribed on a N-BK7 glass substrate with broadband anti-reflective coating on both sides by a tightly focused pulsed femtosecond Bessel beam [22]. The Bessel beam is generated using an ultraviolet fused silica axicon with an apex angle of $179^{\circ}$ and illuminating it with a collimated Gaussian intensity profile pulsed (200 fs) laser beam with a diameter of $2 w=5.3 \mathrm{~mm}$ (at $\left.1 / e^{2}\right), 1030 \mathrm{~nm}$ wavelength, pulse repetition rate of $25 \mathrm{kHz}$, and $8 \mu \mathrm{J}$ pulse energy. The geometry of the fabricated $\mathrm{PhC}$ structures is characterized by the transverse and longitudinal lattice constants $d_{\perp}$ and $d_{\|}$, respectively. The PhC used in our case is a longitudinally chirped structure, meaning that the period along the beam propagation direction $(z)$ is linearly varied. We characterized our structure by defining the geometry factor $Q=2 d_{\perp}^{2} n / \lambda d_{\|}$, which is related to the filtering angle $\sin \alpha=\lambda(Q-1) / 2 d_{\perp} n$. The Bessel beam fabricated two-dimensional $\mathrm{PhC}$ structure has a transverse period $d_{\perp}=3 \mu \mathrm{m}$ and is chirped along the $z$-direction in the range of $1.10 \leq Q \leq 1.60$ with a number of periods $N_{p}=60$ and an aperture of $2.85 \times 2.3 \mathrm{~mm}^{2}$. The PhC fabrication process and the fabricated structure are illustrated in Fig. 5.

Before testing the $\mathrm{PhC}$ inside the resonator, we measured its angular transmission. These measurements are compared with the numerical results using the same parameters. The numerical model uses the split-step method, calculates scattering on a phase mask (corresponding to the refractive-index-modulated
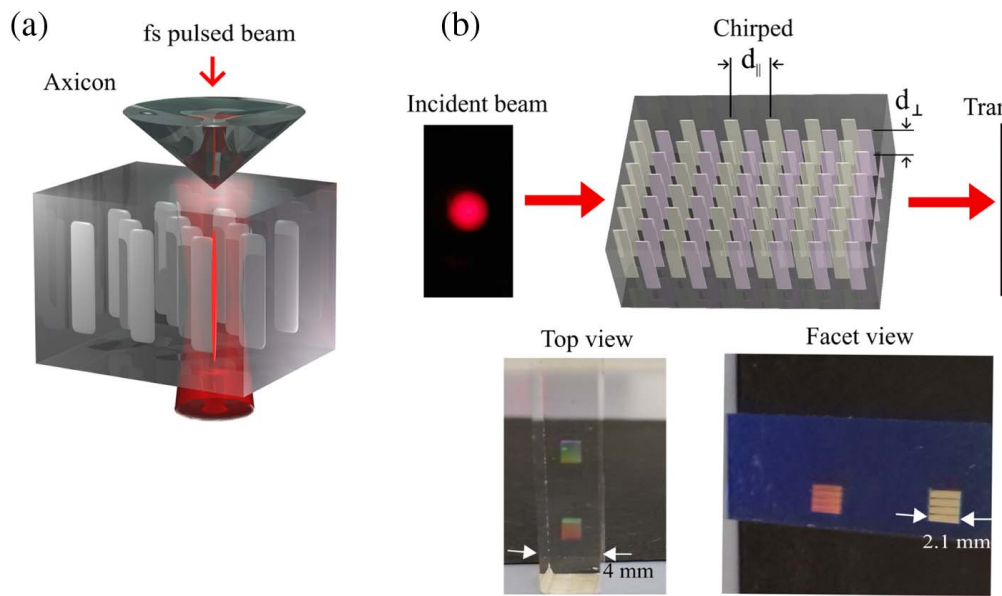

Fig. 5. (a) Simplified schematics illustrating the PhC fabrication using the pulsed Bessel beams. The crystal is fabricated in a glass substrate $(n=$ 1.52) illuminating along the vertical $y$-direction and scanning along the $z$-direction. (b) Top and facet view of fabricated chirped structure illustrating the geometry [14]. Such a structure spatially filters the Gaussian incident probe beam incident from the left and propagating along the $z$-direction. 

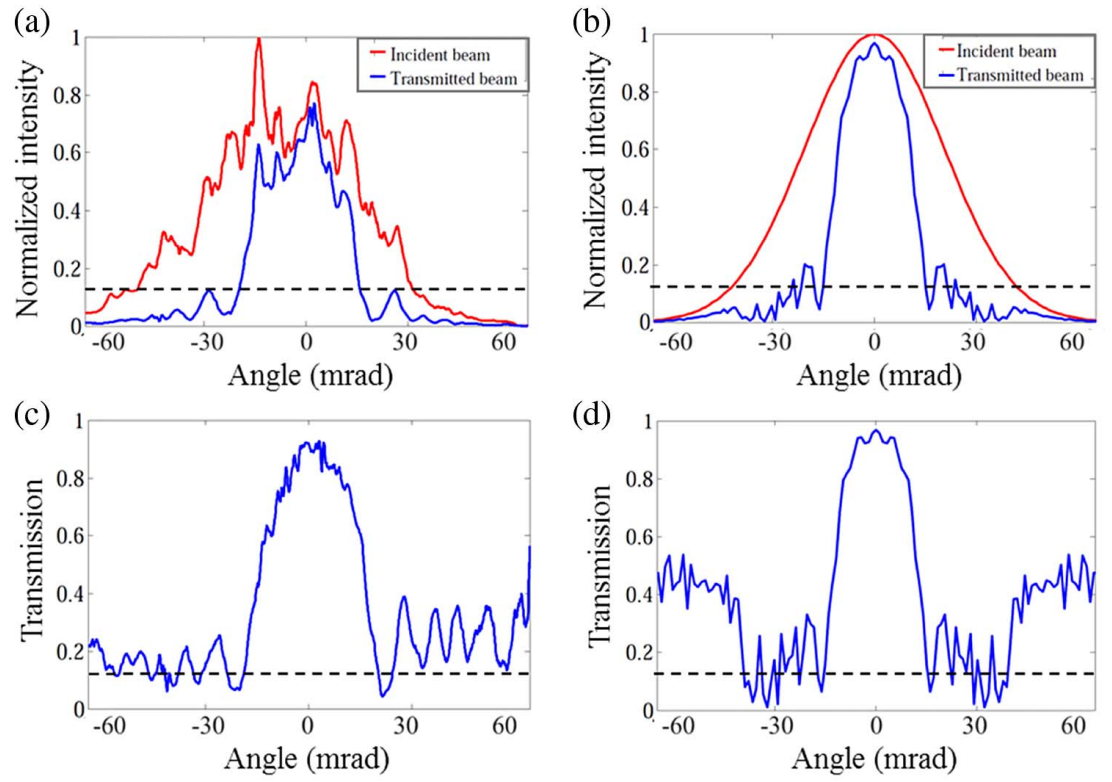

Fig. 6. Single-pass field profiles: (a) experimental measurement with incident beam and transmitted beam through the PhC (b) numerical prediction, Gaussian incident beam, and corresponding transmitted beam by a $\mathrm{PhC}$ with the same parameters as the experimental one. Transmission functions obtained by dividing the transmitted and incident beams for: (c) experimental measurement and (d) numerical simulation of the PhC. The dashed horizontal lines correspond to the $1 / e^{2}$ value.

layer, and diffractive propagation between the layers). The propagation over the crystal is simply the multiplication of matrices of scattering and propagation [23]. The parameters used for this numerical study were $(1.10 \leq Q \leq 1.60$, $\left.N_{p}=60\right)$. The measured and calculated transmission profiles are shown in Fig. 6.

\section{INTRACAVITY PhC SPATIAL FILTERING}

The $\mathrm{PhC}$ is placed inside the cavity in the accessible near-field plane, plane B in Fig. 1(c), at the focal plane of lens $2\left(\mathrm{~L}_{2}\right)$. The field at this plane is an image 1:1 of the field at the front facet of the emitter. The $\mathrm{PhC}$ is mounted on a three-axis stage and carefully arranged inside the cavity. The beam width (secondmoment width or $\mathrm{D} 4 \sigma$ ) at this plane along the slow axis is measured to be $450 \mu \mathrm{m}$, which allows entering the whole beam inside the crystal. Aside, we simulate the $\mathrm{PhC}$ spatial filtering with the same experimental conditions obtaining a good agreement for both, with and without $\mathrm{PhC}$. The chirped $\mathrm{PhC}$ is introduced in numerical simulations as a transmission function in the near field, emulating a compact edge-emitting laser with an integrated $\mathrm{PhC}$, for both counter-propagating fields, $A^{+}$and $A^{-}$. The considered transmission function is the one extracted from the experimental measurements shown in Fig. 6(c). The experimental and numerical far-field distribution with and without $\mathrm{PhC}$ are provided in Figs. 7(a) and 7(b), respectively. We observe a clear correspondence between experimental and numerical field profiles, cutoff angles, and corresponding divergences. We characterized the output beam by measuring the $M^{2}$ value and brightness and studied the filtering performance of the $\mathrm{PhC}$ as a function of the pump current. The pump current is normalized to threshold pump current and is varied from
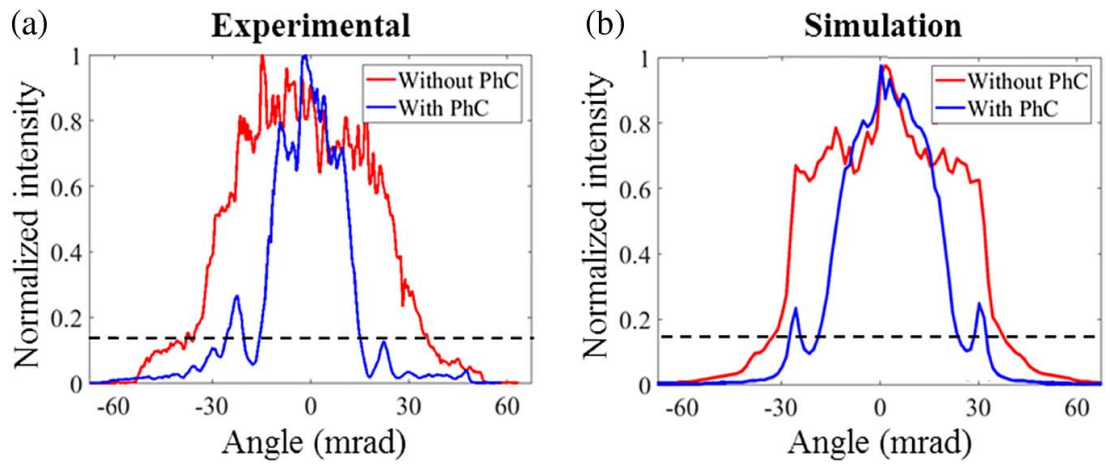

Fig. 7. Effect of the chirped $\mathrm{PhC}$ spatial filtering on the far-field profiles. Comparison of the experimental results (left) versus numerical simulations (right). (a)/(b) Experimental/numerical far-field beam profile. The red curves correspond to the original field, while the blue curves show PhC spatial-filtering beam profile. A clear agreement between the experimental and numerical results was observed. Integration parameters: laser width $=$ $400 \mu \mathrm{m}$, laser length $=1500 \mu \mathrm{m}, \alpha=0.1 \mu \mathrm{m}^{-1}, h=2.0, s=0.04 \mu \mathrm{m}^{-1}, k_{0}=2 \mu \mathrm{m}^{-1}, D=0.03 \mathrm{~cm}^{2} / \mathrm{s}$, and $n=3$. The dashed horizontal lines correspond to the $1 / e^{2}$ value. 
(a)

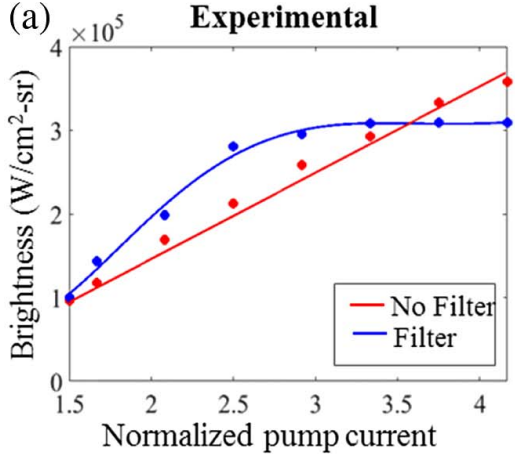

(c)

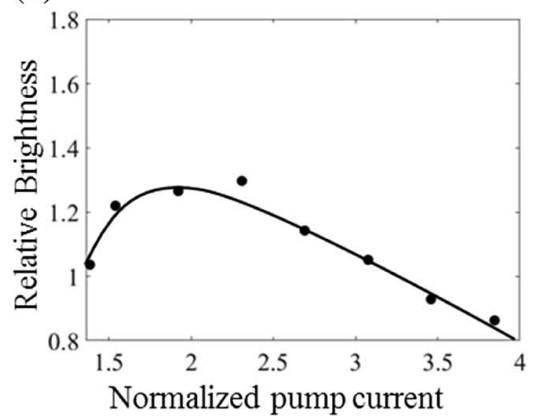

(b)

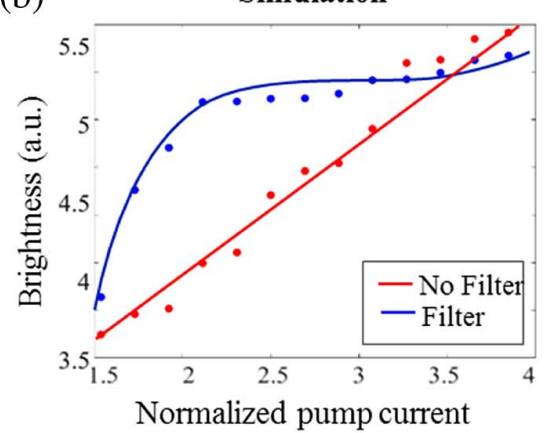

(d)

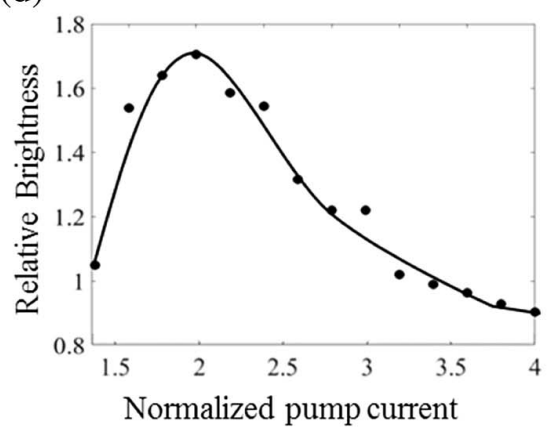

Fig. 8. Brightness and relative brightness, with and without the chirped $\mathrm{PhC}$, as a function of the normalized pump current ranging from 1.5 to 4 . Comparison of the experimental results (left column) versus numerical simulations (right column). (a)/(b) Experimental/simulated brightness. The red lines correspond to the brightness in the unfiltered case. (c)/(d) Experimental/simulated relative brightness. The same transmission profile is used on both experimental and numerical (taken from fig. 6). Integration parameters are as in Fig. 6. The lines are a guide for the eye.

1.5 to 4 . The $M_{x}^{2}$ reduces from 47 to 25 at a normalized pump 2.3 where output power loss is minimum. At higher pump current, we observed reduction in $M_{x}^{2}$ with loss in the output power. While the brightness of the unfiltered laser increases linearly with pump current, as shown by the straight (red) lines in Figs. 8(a) and 8(b), the brightness with the $\mathrm{PhC}$ filtering action initially increases and then stabilizes for larger pump values. The maximum in the absolute brightness is observed at normalized pump currents around 2.5 and remains at similar values as we further increase the pump current. Simulations show a stronger brightness enhancement around a normalized pump current value of two. The plots of the relative brightness clearly show a maximum enhancement brightness of $30 \%$ at a normalized pump current around 2.3 for experiments whereas for simulations, a $70 \%$ maximum enhancement is obtained for a normalized pump of 2, see Figs. 8(c) and 8(d). The smaller pump current dependence in experiments can be attributed to thermal effects inside the cavity that restrict the filtering of higher-order modes whereas this effect is not considered in simulations.

\section{NUMERICAL OPTIMIZATION OF THE FILTERING}

The brightness enhancement is the main motivation of this work which can be further improved in simulations by optimizing the $\mathrm{PhC}$ as well as modifying the intracavity filtering scheme.

Spatial filters based on slits provide extremely convenient transmission functions due to the total extinction of high spatial orders while central modes experience no losses. We optimize the intracavity chirped $\mathrm{PhC}$ by adjusting the main parameters, i.e., the geometry factor $Q$, the chirping (range of $Q_{1}<Q<Q_{2}$ along the $\left.\mathrm{PhC}\right)$, and the number of periods $\left(N_{p}\right)$ to obtain a transmission function approaching that of the slit. Additionally, the $\mathrm{PhC}$ transmission function can be softened to promote intracavity beams approaching Gaussian profiles with smaller $M^{2}$ values, see Fig. 9(a) and the inset of Fig. 9(b). Relative brightness enhancements of $150 \%$ are achieved for a normalized pump current about 2, see Fig. 9(b).

Although up to here we considered a single intracavity filter, i.e., $\mathrm{PhC}$, located between the output facet and the front mirror as in Fig. 10(b), or, equivalently, a slit located at the corresponding far-field plane, other feasible schemes can be numerically studied as well. Next, we consider different configurations where the PhC is located either at the rear mirror, see Fig. 10(a), front mirror as in Fig. 10(b) or simultaneously at both mirrors as on Fig. 10(c); Fig. 10(d) provides the comparison of the brightness enhancements for the optimized $\mathrm{PhC}$ of Fig. 9, from all these configurations, and a slit with various apertures of Fig. 9.

We observed that schemes with spatial filtering at the rear mirror always show smaller relative brightness than those with spatial filtering at the front mirror, while simultaneous filtering at both mirrors achieves the highest relative brightness. Slightly smaller values are attained for the optimized $\mathrm{PhC}$ located at the front and rear mirror while simultaneous $\mathrm{PhCs}$ at both mirrors reaches the maximum relative brightness enhancement of $180 \%$ even higher than the slit case. 

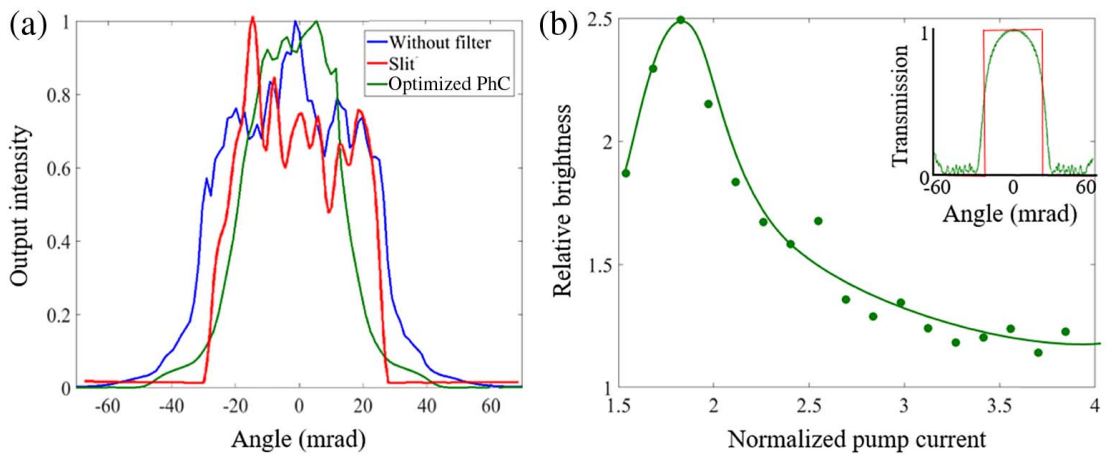

Fig. 9. (a) Output beam profile for the optimized chirped $\mathrm{PhC}$ (green), slit (red) and no intracavity filter (blue). (b) Relative brightness for the optimized $\mathrm{PhC}$ as a function of the normalized pump current, ranging from 1.5 to 4 . The inset in (b) provides the comparison for the transmission function for the optimized $\mathrm{PhC}$ with parameters $Q=1.14, d Q=0.25$, and $N p=73$ (green curve) and the transmission function of a $25 \mathrm{mrad}$ slit (red curve). The rest of numerical parameters are the same as in previous figure. The lines are a guide for the eye.
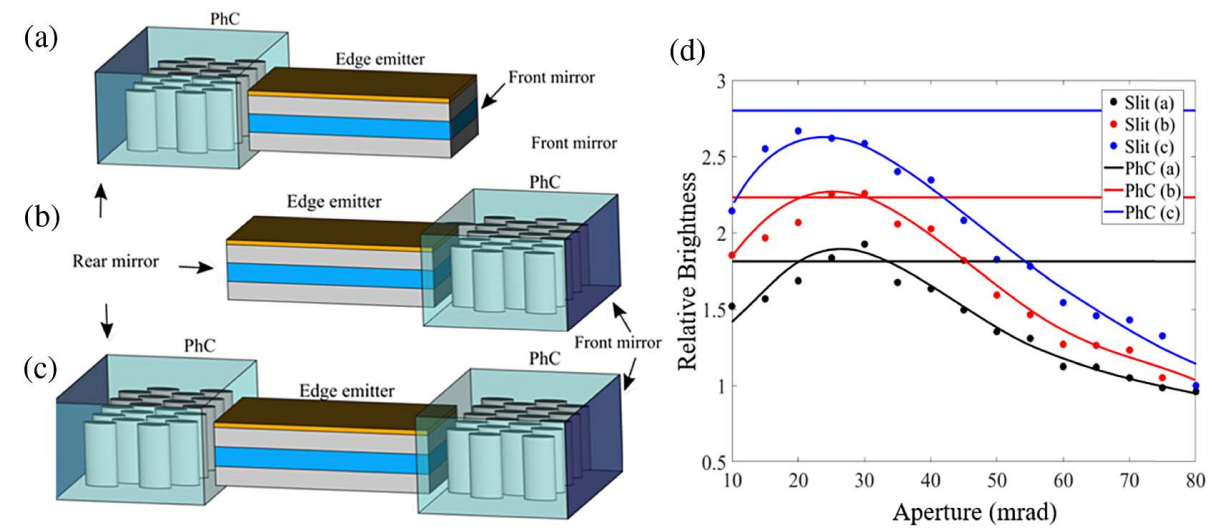

Fig. 10. Scheme of an integrated compact cavity configuration, with the filtering element (in the figures a PhC) at: (a) rear facet, (b) front facet, and (c) both facets. (d) Optimized filtering performance as compared to the slit. The figure shows the relative brightness as a function of the slit aperture for a pump current of $p_{0}=2.5$. The horizontal lines correspond to the chirped PhC filtering results for the proposed configurations: (a) in black, (b) in red, and (c) in blue. The rest of parameters are the same of Fig. 6. The lines are a guide for the eye.

\section{CONCLUSIONS}

We demonstrate spatial filtering in edge-emitting semiconductor lasers both experimentally and numerically. Experiments were performed in an extended cavity configuration using an intracavity two-dimensional chirped $\mathrm{PhC}$ at the near-field plane and comparing it to a variable width slit in the far-field plane. The PhC filtering scheme is advantageous as it allows the miniaturization of the filtering system within a compact laser configuration. We determine that using the $\mathrm{PhC}$, the beam quality parameter $M_{x}^{2}$ is reduced by a factor of 1.8 , bringing along a brightness increase by a factor of 1.3 . The experimental results are in good agreement with the numerical simulations provided by a spatio-temporal model of the compact edge-emitting laser. Moreover, we numerically optimize the intracavity chirped $\mathrm{PhC}$ by approaching its transmission function to the one of a slit. For such an optimized $\mathrm{PhC}$, we numerically achieve an enhancement of the relative brightness over $200 \%$ showing it may be even higher than the filtering performance of a slit. Further improvement may be achieved by exploring different spatialfiltering schemes using the $\mathrm{PhC}$ by placing it at both mirrors. These findings demonstrate that intracavity $\mathrm{PhC}$ filtering may render edge-emitting lasers bright light sources while keeping their compactness.
Funding. Eurostars Project (E-10524 HIP-Lasers); Research Council of Lithuania (09.3.3-LMT-K712-17-0016, SMIP-17-109); Spanish Ministerio de Ciencia e Innovación (PID2019-109175GB-C21).

Acknowledgment. This work has received funding from European Social Fund (project No 09.3.3-LMT-K712-170016) under grant agreement with the Research Council of Lithuania (LMTLT), by the EUROSTARS Project E-10524 HIP-Lasers, as well as by Spanish Ministerio de Ciencia e Innovación, and European Union FEDER through project PID2019-109175GB-C21. D.G. and V.P. acknowledge the financial support from "FOKRILAS" (S-MIP-17-109) from Research Council of Lithuania.

Disclosures. The authors declare no conflicts of interest.

${ }^{\dagger}$ These authors contributed equally to this work.

\section{REFERENCES}

1. H. Sun, A Practical Guide to Handling Laser Diode Beams (Springer, 2015). 
2. S. Zhouping, L. Qihong, D. Jingxing, Z. Jun, and W. Runrong, "Beam quality improvement of laser diode array by using off-axis external cavity," Opt. Express 15, 11776-11780 (2007).

3. E. Samsøe and P. E. Andersen, "Improvement of spatial and temporal coherence of a broad area laser diode using an external-cavity design with double grating feedback," Opt. Express 12, 609-616 (2004).

4. V. Raab and R. Menzel, "External resonator design for high-power laser diode that yields $400 \mathrm{~mW}$ of TEM00 power," Opt. Lett. 27, 167-169 (2002).

5. X. Gao, Y. Zheng, H. Kan, and K. Shinoda, "Effective suppression of beam divergence for high-power laser diode bar by an external-cavity technique," Opt. Lett. 29, 361-363 (2004).

6. M. Mikulla, A. Schmitt, P. Chazan, A. Wetzel, M. Waither, R. Kiefer, W. Pletschen, J. Braunstein, and G. Weimann, "Improved beam quality for high power tapered laser diodes with LMG (low modal gain)-epitaxial layer structures," Proc. SPIE 3284, 72-79 (1998).

7. J. P. Leidner and J. R. Marciante, "Beam quality improvement in broad-area semiconductor lasers via evanescent spatial filtering," IEEE J. Quantum Electron. 48, 1269-1274 (2012).

8. V. V. Popovichev, E. I. Davydova, A. A. Marmalyuk, A. V. Simakov, M. B. Uspenskii, A. A. Chel'nyi, A. P. Bogatov, A. E. Drakin, S. A. Plisyuk, and A. A. Stratonnikov, "High-power single-transverse-mode ridge optical waveguide semiconductor lasers," Quantum Electron. 32 1099-1104 (2002).

9. R. Herrero, M. Botey, M. Radziunas, and K. Staliunas, "Beam shaping in spatially modulated broad-area semiconductor amplifiers," Opt. Lett. 37, 5253 (2012).

10. K. Hirose, Y. Liang, Y. Kurosaka, A. Watanabe, T. Sugiyama, and S. Noda, "Watt-class high-power, high-beam-quality," Nat. Photonics 8, 406-411 (2014).

11. K. Staliunas and V. J. Sánchez-Morcillo, "Spatial filtering of light by chirped photonic crystals," Phys. Rev. A 79, 053807 (2009).

12. Z. Luo, Z. Tang, Y. Xiang, H. Luo, and S. Wen, "Polarizationindependent low-pass spatial filters based on one-dimensional photonic crystals containing negative-index materials," Appl. Phys. B 94, 641-646 (2009).
13. L. Maigyte, T. Gertus, M. Peckus, J. Trull, C. Cojocaru, V. Sirutkaitis, and K. Staliunas, "Signatures of light-beam spatial filtering in a threedimensional photonic crystal," Phys. Rev. A 82, 043819 (2010).

14. S. Gawali, D. Gailevičius, G. Garre-Werner, V. Purlys, C. Cojocaru, J. Trull, J. Montiel-Ponsoda, and K. Staliunas, "Photonic crystal spatial filtering in broad aperture diode laser," Appl. Phys. Lett. 115, 141104 (2019).

15. G. P. Agrawal, "Fast-Fourier-transform based beam-propagation model for stripe-geometry semiconductor lasers: inclusion of axial effects," J. Appl. Phys. 56, 3100-3109 (1984).

16. E. A. Ultanir, D. Michaelis, F. Lederer, and G. I. Stegeman, "Stable spatial solitons in semiconductor optical amplifiers," Opt. Lett. 28, 251-253 (2003).

17. M. Radziunas, J. Fuhrmann, A. Zeghuzi, H. J. Wünsche, T. Koprucki, C. Brée, H. Wenzel, and U. Bandelow, "Efficient coupling of dynamic electro-optical and heat-transport models for high-power broad-area semiconductor lasers,” Opt. Quantum Electron. 51, 69 (2019).

18. J. Medina, R. Herrero, M. Botey, and K. Staliunas, "Stabilized narrowbeam emission from broad-area semiconductor lasers," Phys. Rev. A 101, 033833 (2020).

19. J. M. Buldú, F. Rogister, J. Trull, C. Serrat, M. C. Torrent, C. R. Mirasso, and J. Garcia-Ojalvo, "Dynamics of power distribution in multimode semiconductor lasers with optical feedback," Proc. SPIE 4646, 411-419 (2002).

20. B. Leonhäuser, H. Kissel, A. Unger, B. Köhler, and J. Biesenbach, "Feedback-induced catastrophic optical mirror damage (COMD) on $976 \mathrm{~nm}$ broad area single emitters with different AR reflectivity," Proc. SPIE 8965, 896506 (2014).

21. C. Holly, S. Hengesbach, M. Traub, and D. Hoffmann, "Simulation of spectral stabilization of high-power broad-area edge emitting semiconductor lasers," Opt. Express 21, 15553-15567 (2013).

22. D. Gailevičius, V. Purlys, and K. Staliunas, "Photonic crystal spatial filters fabricated by femtoseconds pulsed Bessel beam," Opt. Lett. 44, 4969-4972 (2019).

23. L. Maigyte and K. Staliunas, "Spatial filtering with photonic crystals," Appl. Phys. Rev. 2, 011102 (2015). 